BARI-TH/08-593

\title{
Light scalar mesons in the soft-wall model of AdS/QCD
}

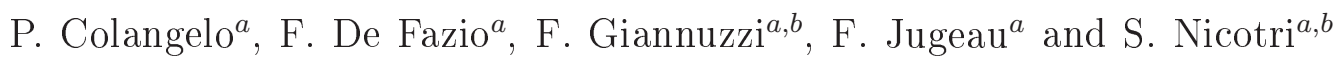 \\ a Istituto Nazionale di Fisica Nucleare, \\ Sezione di Bari, Italy \\ b Dipartimento di Fisica, \\ Università di Bari, Italy
}

\begin{abstract}
We study light scalar mesons in the AdS/QCD soft-wall model with a background dilaton field. The masses and decay constants are compatible with experiment and QCD determinations if $a_{0}(980)$ and $f_{0}(980)$ are identified as the lightest scalar mesons; moreover, the states are organized in linear Regge trajectories with the same slope of vector mesons. Comparing the two-point correlation function of scalar operators in AdS and QCD, information about the condensates can be derived. Strong couplings of scalar states to pairs of light pseudoscalar mesons turn out to be small, at odds with experiment and QCD estimates: this discrepancy is related to the description of chiral symmetry breaking in this model.

PACS numbers: $11.25 . \mathrm{Tq}, 12.39 . \mathrm{Mk}, 12.90 .+\mathrm{b}$
\end{abstract}




\section{INTRODUCTION}

The idea of extending the AdS/CFT correspondence conjecture [1] to QCD-like theories [2] has provided new hints on the possibility of describing strong interaction processes by string-inspired approaches. Two main ways have been followed to achieve such a result. The first, the so-called top-down approach, consists in starting from a string/M-theory living on $\operatorname{AdS}_{d+1} \times \mathcal{C}(\mathcal{C}$ being a compact manifold $)$ and attempting a derivation of a low-energy QCDlike theory on the flat boundary $\mathcal{M}_{d}$ of the AdS space through appropriate compactifications of the extra dimensions [2, 3]. In the second one, the so-called bottom-up approach, one starts from $4 d$ QCD and attempts to construct its higher dimensional dual theory (not necessarily a string one), assuming its existence [4], with phenomenological properties as guidelines.

An important aspect of both these approaches is the necessity of a mechanism to break conformal invariance, since QCD is not a conformal theory [5], and to account for phenomena such as confinement. The way to do this usually consists in incorporating in the dual theory a mass scale related to the QCD scale $\Lambda_{Q C D}$. For example, in the bottom-up approach, one possibility is to use a five dimensional "AdS-slice" letting the fifth (holographic) coordinate $z$ vary in a range up to $z_{\max }$ of $\mathcal{O}\left(\frac{1}{\Lambda_{Q C D}}\right)[4,6,7]$. In this (so-called hard-wall) model, several QCD aspects have been investigated, namely high-energy hadron scattering amplitudes, spectra, form factors, strong couplings, light-front wave functions, Wilson loop [4, 6-9].

Another proposal to break conformal invariance consists in introducing in the $5 d$ AdS holographic space a background dilaton field (the so-called soft-wall model) [10, 11]. While in top-down approaches the dilaton profile must be a solution of the supergravity equations of motion, in this kind of approaches its functional form is chosen on the basis of phenomenological information, namely imposing the Regge behaviour for vector mesons; noticeably, the obtained dilaton profile, found using heuristic arguments, can be justified constructing a suitable dynamical model [12]. Also in this framework many QCD properties have been investigated, such as vector and tensor meson masses and form factors, glueball masses, the static $\bar{Q} Q$ potential and DIS [10, 11, 13-17]. The results of the two bottom-up models differ in many respects; the possibility of continuously interpolating between them has also been considered [18], trying to recognize the essential features of the QCD dual.

Since light scalar mesons represent an important and debated sector of QCD, it is in- 
teresting to consider them in the holographic framework, and indeed some analyses have been carried out $[19,20]$. Here we study the scalar sector in the soft-wall model, trying to identify which properties can be described in the holographic approach. In particular, we consider the mass spectrum, the decay constants and the strong couplings of scalar mesons to pairs of light pseudoscalars. The comparison of the results obtained in the AdS framework with experiment and QCD calculations can shed light on the features and drawbacks of this model.

\section{THE MODEL}

The model we investigate is defined in the $5 d$ space with metric:

$$
d s^{2}=g_{M N} d x^{M} d x^{N}=\frac{R^{2}}{z^{2}}\left(\eta_{\mu \nu} d x^{\mu} d x^{\nu}+d z^{2}\right)
$$

with $\eta_{\mu \nu}=\operatorname{diag}(-1,+1,+1,+1) ; R$ is the AdS curvature radius and the coordinate $z$ runs in the range $0 \leq z<+\infty$ (or, considering a UV cutoff, from the ultra-violet brane $z_{\min }=\epsilon$ to $+\infty)$.

In addition to the AdS metric, the model is characterized by a background dilaton field:

$$
\Phi(z)=(c z)^{2}
$$

the form of which is chosen to obtain light vector mesons with linear Regge trajectories [10]; $c$ is a dimensionful parameter setting the scale of QCD quantities.

We consider the $5 d$ action:

$$
S_{e f f}=-\frac{1}{k} \int d^{5} x \sqrt{-g} e^{-\Phi(z)} \operatorname{Tr}\left\{|D X|^{2}+m_{5}^{2} X^{2}+\frac{1}{4 g_{5}^{2}}\left(F_{L}^{2}+F_{R}^{2}\right)\right\}
$$

where $g$ is the determinant of the metric tensor $g_{M N}$ in (1) and $\Phi$ the background dilaton field (2). This action includes fields which are dual to QCD operators defined at the boundary $z=0$. There is a scalar bulk field $X$, the mass of which is fixed by the AdS/CFT relation: $m_{5}^{2} R^{2}=(\Delta-p)(\Delta+p-4), \Delta$ being the dimension of the $p$-form QCD operator dual to $X$. This field, written as

$$
X=\left(X_{0}+S\right) e^{2 i \pi}
$$

contains a background field $X_{0}(z)=\frac{v(z)}{2}$, the scalar field $S(x, z)$ and the chiral field $\pi(x, z)$. $X_{0}$ only depends on $z$ and is dual to $\langle\bar{q} q\rangle$; since it is different from zero, it represents the 
term responsible for the breaking of chiral symmetry. The scalar bulk field $S$ includes singlet $S_{1}(x, z)$ and octet $S_{8}^{a}(x, z)$ components, gathered into the multiplet:

$$
S=S^{A} T^{A}=S_{1} T^{0}+S_{8}^{a} T^{a}
$$

with $T^{0}=1 / \sqrt{2 n_{F}}=1 / \sqrt{6}$ and $T^{a}$ the generators of $S U(3)_{F}$, with normalization

$$
\operatorname{Tr}\left(T^{A} T^{B}\right)=\frac{\delta^{A B}}{2}
$$

$(A=0, a$, and $a=1, \ldots 8) . S^{A}$ is dual to the QCD operator $\mathcal{O}_{S}^{A}(x)=\bar{q}(x) T^{A} q(x)$, so that $\Delta=3, p=0$ and $m_{5}^{2} R^{2}=-3$. The fact that the scalar bulk field is tachyonic does not affect the stability of the theory, since fields with slightly negative masses are allowed, as discussed in [21].

The action (3) also involves the fields $A_{L, R}^{a}(x, z)$ introduced to gauge the chiral symmetry in the $5 d$ space. They are dual to the QCD operators $\bar{q}_{L, R} \gamma_{\mu} T^{a} q_{L, R}$, with field strengths

$$
F_{L, R}^{M N}=F_{L, R}^{M N a} T^{a}=\partial^{M} A_{L, R}^{N}-\partial^{N} A_{L, R}^{M}-i\left[A_{L, R}^{M}, A_{L, R}^{N}\right]
$$

The gauge fields enter in the covariant derivative: $D^{M} X=\partial^{M} X-i A_{L}^{M} X+i X A_{R}^{M}$. Writing $A_{L, R}$ in terms of vector $V$ and axial-vector $A$ fields: $V^{M}=\frac{1}{2}\left(A_{L}^{M}+A_{R}^{M}\right)$ and $A^{M}=\frac{1}{2}\left(A_{L}^{M}-\right.$ $\left.A_{R}^{M}\right)$, we obtain the action:

$$
S_{e f f}=-\frac{1}{k} \int d^{5} x \sqrt{-g} e^{-\Phi(z)} \operatorname{Tr}\left\{|D X|^{2}+m_{5}^{2} X^{2}+\frac{1}{2 g_{5}^{2}}\left(F_{V}^{2}+F_{A}^{2}\right)\right\}
$$

with

$$
\begin{aligned}
& F_{V}^{M N}=\partial^{M} V^{N}-\partial^{N} V^{M}-i\left[V^{M}, V^{N}\right]-i\left[A^{M}, A^{N}\right] \\
& F_{A}^{M N}=\partial^{M} A^{N}-\partial^{N} A^{M}-i\left[V^{M}, A^{N}\right]-i\left[A^{M}, V^{N}\right]
\end{aligned}
$$

and $D^{M} X=\partial^{M} X-i\left[V^{M}, X\right]-i\left\{A^{M}, X\right\}$.

The action (3)-(8) is the starting point of our analysis. Following the AdS/CFT guideline, we assume that the duality relation holds:

$$
\left\langle e^{i \int d^{4} x \mathcal{O}(x) f_{0}(x)}\right\rangle_{Q C D}=e^{i S_{e f f}}
$$

where the lhs is the QCD generating functional in which the sources $f_{0}(x)$ of the $4 d \mathcal{O}(x)$ operators are the boundary $(z \rightarrow 0)$ limits of the corresponding (dual) $5 d$ fields. We then derive the properties of light scalar mesons on the basis of the AdS/CFT duality procedure applied to the soft-wall model. The check of duality in this channel is the aim of the forthcoming Sections. 


\section{SPECTRUM OF SCALAR MESONS}

Let us consider the quadratic part of the action (3)-(8) involving the scalar fields $S^{A}(x, z)$ :

$$
S_{e f f}^{(2)}=-\frac{1}{2 k} \int d^{5} x \sqrt{-g} e^{-\Phi(z)}\left(g^{M N} \partial_{M} S^{A} \partial_{N} S^{A}+m_{5}^{2} S^{A} S^{A}\right) .
$$

From this term, it is straightforward to derive the equation of motion for the field $S^{A}$ (for any flavour index $A$, which is dropped below):

$$
\eta^{M N} \partial_{M}\left(\frac{R^{3}}{z^{3}} e^{-\Phi(z)} \partial_{N} S\right)+3 \frac{R^{3}}{z^{5}} e^{-\Phi(z)} S=0
$$

or, in the $4 d$ Fourier space, defining $S(x, z)=\int \frac{d^{4} q}{(2 \pi)^{4}} e^{i q \cdot x} \tilde{S}(q, z)$ (from now on the tilde will always denote $4 d$ Fourier-transformed fields):

$$
\partial_{z}\left(\frac{R^{3}}{z^{3}} e^{-\Phi(z)} \partial_{z} \tilde{S}\right)+3 \frac{R^{3}}{z^{5}} e^{-\Phi(z)} \tilde{S}-q^{2} \frac{R^{3}}{z^{3}} e^{-\Phi(z)} \tilde{S}=0
$$

Scalar meson states correspond to the normalizable solutions of this equation. The solutions can be obtained considering the transformation:

$$
\tilde{S}=e^{\left(\hat{z}^{2}+3 \log \hat{z}\right) / 2} Y
$$

with $\hat{z}=c z$ and the function $Y$ satisfying the one dimensional Schrödinger-like equation

$$
-Y^{\prime \prime}+V(\hat{z}) Y=\frac{m^{2}}{c^{2}} Y
$$

the derivatives act on $\hat{z}$, and the potential is $V(\hat{z})=\hat{z}^{2}+\frac{3}{4 \hat{z}^{2}}+2$. The normalizable solutions of eq.(15) correspond to the discrete mass spectrum [20]:

$$
-q_{n}^{2}=m_{n}^{2}=c^{2}(4 n+6)
$$

with integer $n$, and eigenfunctions expressed in terms of the generalized Laguerre polynomials:

$$
\tilde{S}_{n}(\hat{z})=\sqrt{\frac{2}{n+1}} \hat{z}^{3} L_{n}^{1}\left(\hat{z}^{2}\right) .
$$

The results of this simple calculation can be compared to current phenomenology. Scalar mesons are organized in linear Regge trajectories, as a consequence of the choice of the dilaton field (2). The slope of the trajectories is the same as for vector mesons, the spectral condition of which is [10]:

$$
m_{\rho_{n}}^{2}=c^{2}(4 n+4)
$$


In the same soft-wall model also scalar glueballs appear in Regge trajectories with the same slope, since their masses are given by [16]:

$$
m_{G_{n}}^{2}=c^{2}(4 n+8)
$$

therefore the parameter $c$ sets the scale of all hadron masses.

Scalar mesons turn out to be heavier than vector mesons. This is in agreement with experiment if $a_{0}(980)$ and $f_{0}(980)$ are identified as the lightest scalar mesons. The agreement is quantitative, since eqs.(16) and (18) allow to predict: $R_{f_{0}\left(a_{0}\right)}=\frac{m_{f_{0}\left(a_{0}\right)}^{2}}{m_{\rho^{0}}^{2}}=\frac{3}{2}$, to be compared to $R_{f_{0}}^{e x p}=1.597 \pm 0.033$ and $R_{a_{0}}^{e x p}=1.612 \pm 0.004$. Considering the first radial exitations, the predictions $R_{f_{0}\left(a_{0}\right)}^{\prime}=\frac{5}{4}$ should be compared to the measurements $R_{f_{0}}^{\text {exp }}=1.06 \pm 0.04$ and $R_{a_{0}}^{\text {exp }}=1.01 \pm 0.04$, having identified $a_{0}(1450), f_{0}(1505)$ and $\rho(1450)$ as radial excitations; an assignment which however could be questionable in case of $f_{0}(1505)$ (identifying $f_{0}(1370)$ with the first radial excitation, one finds $R_{f_{0}}^{\text {exp }}=0.9 \pm 0.2$ ).

Finally, scalar mesons are lighter than scalar glueballs: $\frac{m_{G}^{2}}{m_{f_{0}}^{2}}=\frac{4}{3}$ for the lowest-lying states. Hierarchy among the hadron species is reduced for higher radial states, which become degenerate when the quantum number $n$ increases.

\section{BULK-TO-BOUNDARY PROPAGATOR OF THE SCALAR FIELD}

According to the AdS/CFT correspondence, the value of the $5 d$ field $\widetilde{S}\left(q^{2}, z\right)$ at the UV boundary $z=0$, i.e. $\widetilde{S}_{0}\left(q^{2}\right)$, acts as the source of the corresponding dual $4 d$ operator in the QCD functional integral. They are related through the bulk-to-boundary propagator: $\widetilde{S}\left(q^{2}, z\right)=S\left(q^{2} / c^{2}, \hat{z}^{2}\right) \widetilde{S}_{0}\left(q^{2}\right)$. This propagator is obtained solving, for all values of the four-momenta $q^{2}$, eq.(13) which can be cast in the form:

$$
S^{\prime \prime}-\frac{1}{\hat{z}}\left(2 \hat{z}^{2}+3\right) S^{\prime}-\left(\frac{q^{2}}{c^{2}}-\frac{3}{\hat{z}^{2}}\right) S=0
$$

with the derivatives acting on $\hat{z}$. The general solution of this equation involves the Tricomi confluent hypergeometric function $U$ and the Kummer confluent hypergeometric function ${ }_{1} F_{1}:$

$$
S\left(\frac{q^{2}}{c^{2}}, \hat{z}^{2}\right)=\frac{1}{R c} \Gamma\left(\frac{q^{2}}{4 c^{2}}+\frac{3}{2}\right) \hat{z} U\left(\frac{q^{2}}{4 c^{2}}+\frac{1}{2} ; 0 ; \hat{z}^{2}\right)+B\left(\frac{q^{2}}{c^{2}}\right) \hat{z}^{3}{ }_{1} F_{1}\left(\frac{q^{2}}{4 c^{2}}+\frac{3}{2} ; 2 ; \hat{z}^{2}\right)
$$

with $B\left(\frac{q^{2}}{c^{2}}\right)$ an undetermined function of $q^{2} / c^{2}$. If we impose the boundary condition that the action is finite in the IR region $z \rightarrow+\infty$ (a standard assumption in the soft-wall model 
approach) the solution with $B=0$ must be chosen; we mention below the consequences of relaxing such a condition, as studied in [22] for scalar glueballs. In the UV $z \rightarrow 0$ limit, the boundary condition

$$
S\left(\frac{q^{2}}{c^{2}}, \hat{z}^{2}\right) \underset{z \rightarrow 0}{\rightarrow} \frac{z}{R}
$$

fixes the coefficient of the Tricomi function $U$. With this expression of the bulk-to-boundary propagator it is possible to compute several quantities, namely two- and three-point correlation functions involving scalar operators.

Before continuing with the analysis, it is worth reminding the features of the background field $X_{0}(z)=\frac{v(z)}{2}$. It is solution of the linearized equation of motion:

$$
\partial_{z}\left(\frac{R^{3}}{z^{3}} e^{-\Phi(z)} \partial_{z} v(z)\right)+3 \frac{R^{3}}{z^{5}} e^{-\Phi(z)} v(z)=0
$$

which explicitely reads:

$$
v(z)=\frac{m_{q}}{R c} \Gamma(3 / 2) \hat{z} U\left(1 / 2 ; 0 ; \hat{z}^{2}\right)+C \hat{z}^{3}{ }_{1} F_{1}\left(3 / 2 ; 2 ; \hat{z}^{2}\right)
$$

Since both the Tricomi and the Kummer confluent hypergeometric functions go to unity for $z \rightarrow 0$, the asymptotic UV behaviour of (24) is:

$$
v(z) \underset{z \rightarrow 0}{\rightarrow} \frac{m_{q} z}{R}+\frac{\Sigma z^{3}}{R}
$$

with $\Sigma$ related to the constant $C$. Using the AdS/CFT dictionary, the coefficient of $z$ enters in the (UV) boundary condition related to the quark mass, while the coefficient of the $z^{3}$ term is fixed by the (UV) boundary condition related to the chiral condensate, the two quantities being responsible of chiral symmetry breaking. However, if one imposes as an IR boundary condition that $v(z)$ does not diverge at $z \rightarrow+\infty$, in $(24)$ the solution with $C=0$ must be chosen, so that the low- $z$ expansion of $v$ reads:

$$
v(z) \underset{z \rightarrow 0}{\rightarrow} \frac{m_{q} z}{R}-\frac{c^{2} m_{q}}{2 R}\left(1-2 \gamma_{E}-2 \ln (c z)-\psi(3 / 2)\right) z^{3}
$$

with $\psi$ the Euler function. Identifying the coefficient of the $z^{3}$ term as the chiral condensate, from eq.(26) a proportionality relation can be established between the quark mass and the quark condensate; this kind of relation is absent in QCD. This shortcoming, already recognized in the soft-wall model of AdS/QCD [10], does not appear in the hard-wall model where the coefficients of $z$ and $z^{3}$ terms of $v$ are independent. In principle, it could be avoided 
by adding potential terms $U(X)$ to the action (3), as suggested in [10]; models for $v(z)$ with the asymptotic UV and IR behaviour dictated by (23) have also been investigated [18]. In the following, we ignore this difficulty and use the expression of $v(z)$ in $(24)$ with $C=0$; the consequences are important for the scalar meson couplings to pairs of pseudoscalar states, as we discuss below [23].

Before concluding this Section, we report the equations of motion for the axial and the pion fields. Writing the axial field $\widetilde{A}_{\mu}^{a}$ in terms of its transverse and longitudinal components: $\widetilde{A}_{\mu}^{a}=\widetilde{A}_{\mu \perp}^{a}+i q_{\mu} \widetilde{\phi}^{a}$, we have, from the action (3)-(8):

$$
\begin{aligned}
& {\left[\partial_{z}\left(\frac{e^{-\Phi}}{z} \partial_{z} \widetilde{A}_{\mu}^{a}\right)-\frac{q^{2} e^{-\Phi}}{z} \widetilde{A}_{\mu}^{a}-\frac{g_{5}^{2} R^{2} v(z)^{2} e^{-\Phi}}{z^{3}} \widetilde{A}_{\mu}^{a}\right]_{\perp}=0} \\
& \partial_{z}\left(\frac{e^{-\Phi}}{z} \partial_{z} \widetilde{\phi}^{a}\right)+\frac{g_{5}^{2} R^{2} v(z)^{2} e^{-\Phi}}{z^{3}}\left(\widetilde{\pi}^{a}-\widetilde{\phi}^{a}\right)=0 \\
& q^{2} \partial_{z} \widetilde{\phi}^{a}+\frac{g_{5}^{2} R^{2} v(z)^{2}}{z^{2}} \partial_{z} \widetilde{\pi}^{a}=0 .
\end{aligned}
$$

These equations will be considered below when we compute the scalar meson couplings to pairs of light pseudoscalar states.

\section{TWO-POINT CORRELATION FUNCTION OF THE SCALAR OPERATOR}

Let us consider in QCD the two-point correlation function:

$$
\Pi_{Q C D}^{A B}\left(q^{2}\right)=i \int d^{4} x e^{i q \cdot x}\left\langle 0\left|T\left[\mathcal{O}_{S}^{A}(x) \mathcal{O}_{S}^{B}(0)\right]\right| 0\right\rangle
$$

with $\mathcal{O}_{S}^{A}(x)=\bar{q}(x) T^{A} q(x)$. The AdS/CFT method relates this correlation function to the two-point correlator obtained from the action (3)-(8), which can be written in terms of the bulk-to-boundary propagator (21):

$$
\Pi_{A d S}^{A B}\left(q^{2}\right)=\left.\delta^{A B} \frac{R^{3} c^{4}}{k} S\left(\frac{q^{2}}{c^{2}}, \hat{z}^{2}\right) \frac{e^{-\Phi(\hat{z})}}{\hat{z}^{3}} \partial_{\hat{z}} S\left(\frac{q^{2}}{c^{2}}, \hat{z}^{2}\right)\right|_{\hat{z} \rightarrow 0}
$$

with the result

$$
\begin{aligned}
\Pi_{A d S}^{A B}\left(q^{2}\right) & =\delta^{A B} \frac{4 c^{2} R}{k}\left[\left(\frac{q^{2}}{4 c^{2}}+\frac{1}{2}\right) \ln \left(c^{2} z^{2}\right)+\left(\gamma_{E}-\frac{1}{2}\right)+\frac{q^{2}}{4 c^{2}}\left(2 \gamma_{E}-\frac{1}{2}\right)\right. \\
& \left.+\left(\frac{q^{2}}{4 c^{2}}+\frac{1}{2}\right) \psi\left(\frac{q^{2}}{4 c^{2}}+\frac{3}{2}\right)\right]\left.\right|_{z=z_{\text {min }}}
\end{aligned}
$$




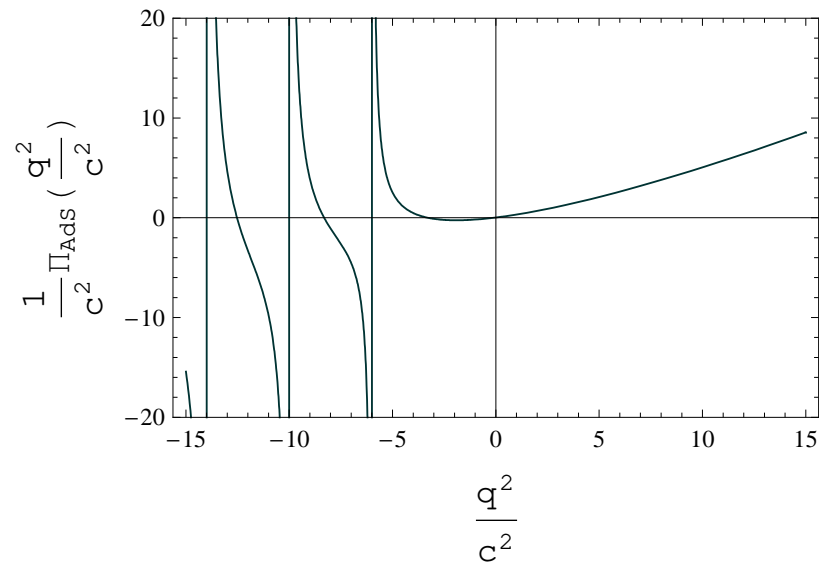

Figure 1: Two-point correlation function $\frac{1}{c^{2}} \Pi_{A d S}\left(q^{2} / c^{2}\right)$ in eq.(32). The renormalization scale is fixed to $\nu=1 \mathrm{GeV}$.

(omitting a $\mathcal{O}\left(\frac{1}{z^{2}}\right)$ contact term). The AdS expression of the correlation function (plotted in fig.1) shows the presence of a discrete set of poles, corresponding to the poles of the Euler function $\psi$, with masses given by the spectral relation (16) and residues

$$
F_{n}^{2}=\frac{R}{k} 16 c^{4}(n+1) .
$$

The factor $\frac{R}{k}$ can be fixed by matching the AdS expression (32) in the $q^{2} \rightarrow+\infty$ (i.e. in the short-distance) limit, expanded in powers of $1 / q^{2}$, with the QCD result. For $\Pi_{A d S}$, identifying $z_{\min }$ with the renormalization scale $\frac{1}{\nu}$, we get:

$$
\begin{aligned}
\Pi_{A d S}^{A B}\left(q^{2}\right) & =\delta^{A B} \frac{R}{k}\left[q^{2} \ln \left(\frac{q^{2}}{\nu^{2}}\right)+q^{2}\left(2 \gamma_{E}-\ln 4-\frac{1}{2}\right)+2 c^{2}\left(\ln \left(\frac{q^{2}}{\nu^{2}}\right)-\ln 4+2 \gamma_{E}+1\right)\right. \\
& \left.+\frac{2}{3} \frac{c^{4}}{q^{2}}+\frac{4}{3} \frac{c^{6}}{q^{4}}+O\left(1 / q^{6}\right)\right]
\end{aligned}
$$

while the QCD result, for $N_{c}=3$ and in terms of quark and gluon condensates, is [24]:

$$
\begin{aligned}
\Pi_{Q C D}^{A B}\left(q^{2}\right) & =\frac{\delta^{A B}}{2}\left[\frac{3}{8 \pi^{2}}\left(1+\frac{11 \alpha_{s}}{3 \pi}\right) q^{2} \ln \left(\frac{q^{2}}{\nu^{2}}\right)+\frac{3}{q^{2}}\left\langle m_{q} \bar{q} q\right\rangle+\frac{1}{8 q^{2}}\left\langle\frac{\alpha_{s}}{\pi} G^{2}\right\rangle\right. \\
& +\frac{m_{q} g_{s}}{2 q^{4}}\left\langle\left(\bar{q} \sigma_{\mu \nu} \lambda^{a} q\right) G_{\mu \nu}^{a}\right\rangle+\frac{\pi \alpha_{s}}{q^{4}}\left\langle\left(\bar{q} \sigma_{\mu \nu} \lambda^{a} q\right)^{2}\right\rangle \\
& \left.+\frac{2 \pi \alpha_{s}}{3 q^{4}}\left\langle\left(\bar{q} \gamma_{\mu} \lambda^{a} q\right) \sum_{q=u, d} \bar{q} \gamma_{\mu} \lambda^{a} q\right\rangle+O\left(1 / q^{6}\right)\right]
\end{aligned}
$$


where possible terms related, e.g., to instanton contributions have not been considered. Matching the perturbative term fixes the condition:

$$
\frac{R}{k}=\frac{N_{c}}{16 \pi^{2}}
$$

In the same way, by the two-point correlation function of the vector current, the matching condition fixes the value of $g_{5}^{2}: g_{5}^{2}=3 / 4$.

The residues of the two-point correlation function, related to the scalar meson decay constants, are now determined:

$$
F_{n}^{2}=\frac{N_{c}}{\pi^{2}} c^{4}(n+1)
$$

for all radial states labeled by $n$.

It is interesting to compare (37) to QCD calculations. For $a_{0}(980)$, the following result has been obtained for the current-vacuum matrix elements defining the decay constants:

$$
F_{a_{0}}=\left\langle 0\left|\mathcal{O}_{S}^{3}\right| a_{0}(980)^{0}\right\rangle=(0.21 \pm 0.05) \mathrm{GeV}^{2}[25]
$$

The AdS prediction is: $F_{a_{0}}=\frac{\sqrt{3}}{\pi} c^{2}=0.08 \mathrm{GeV}^{2}$, having fixed $c$ from the $\rho^{0}$ mass: $c=$ $\frac{m_{\rho}}{2}$. For the $f_{0}(980)$ a similar result has been obtained for the matrix element of the $s \bar{s}$ operator: $\left\langle 0|\bar{s} s| f_{0}(980)\right\rangle=(0.18 \pm 0.015) \mathrm{GeV}^{2}$ [26]. The AdS result is not far from QCD determinations. For the first radial excitation we have: $F_{a_{0}^{\prime}}=0.12 \mathrm{GeV}^{2}$, while for large values of $n$ the ratio $\frac{F_{n}^{2}}{m_{n}^{2}}$ becomes independent of the radial quantum number.

AdS/QCD duality can be checked for the various terms in the $\frac{1}{q^{2}}$ power expansion, comparing eqs.(34) and (35). For $m_{q}=0$, the four dimensional gluon condensate can be computed:

$$
\left\langle\frac{\alpha_{s}}{\pi} G^{2}\right\rangle=\frac{2}{\pi^{2}} c^{4} \simeq 0.004 \mathrm{GeV}^{4}
$$

which is smaller than the commonly used value $\left\langle\frac{\alpha_{s}}{\pi} G^{2}\right\rangle \simeq 0.012 \mathrm{GeV}^{4}$, the estimated uncertainty of which is about $30 \%$ [27].

Considering $O\left(1 / q^{4}\right)$ terms, in QCD one can use the factorization approximation:

$$
\begin{aligned}
\left\langle\left(\bar{q} \sigma_{\mu \nu} \lambda^{a} q\right)^{2}\right\rangle & \simeq-\frac{16}{3}\langle\bar{q} q\rangle^{2}, \\
\left\langle\left(\bar{q} \gamma_{\mu} \lambda^{a} q\right)^{2}\right\rangle & \simeq-\frac{16}{9}\langle\bar{q} q\rangle^{2}
\end{aligned}
$$

for the dimension 6 operators. Within such an approximation, the AdS and QCD expressions do not match, since the $O\left(1 / q^{4}\right)$ term in (34) is positive, while it is negative in (35). 
The last remark is that in the AdS expression (34) there is a contribution interpreted in terms of a dimension two condensate, while an analogous term is absent in the QCD expansion (35). In this respect, the two-point correlation function of the scalar operators presents the same phenomenon occurring in the two-point correlation function of vector mesons [28, 29] and of scalar glueballs [22, 30]. Although in QCD there is no local gaugeinvariant operator of dimension two, the possible relevance of a dimension two condensate in the form of an effective gluon mass term is the subject of discussions [31], so that the AdS result could be interpreted as an argument supporting the existence of this condensate. However, the AdS/CFT method dictates duality between bulk fields and gauge-invariant operators in the boundary theory. Another possible way to explain the presence of this contribution is that, although the quadratic dependence of the dilaton field in the IR is required to provide linear confinement, at smaller values of $z$ the functional dependence of $\Phi(z)$ is less constrained, so that in other versions of the background field such a term could be removed: this deserves an explicit check. A different possibility, put forward in [22], is that the subleading (for $z \rightarrow 0$ ) solution in the bulk-to-boundary scalar field propagator plays a role, so that its coefficient can be tuned to cancel the dimension two contribution. In such a scenario, in which the AdS dual theory needs to be regularized in the IR, the subleading solution modifies some terms in the power expansion of the two-point correlation function, leaving the perturbative term unaffected.

\section{INTERACTION OF SCALAR MESONS WITH A PAIR OF PSEUDOSCALAR MESONS}

In the action (3) the interaction terms involving one scalar $S$ and two light pseudoscalar fields $P$ only appear in the covariant derivative $\operatorname{Tr}\left\{|D X|^{2}\right\}$. Using the equations of motion and writing the axial-vector bulk field in terms of the transverse and longitudinal components: $A_{M}=A_{\perp M}+\partial_{M} \phi$, we have:

$$
S_{e f f}^{(S P P)}=-\frac{4}{k} \int d^{5} x \sqrt{-g} e^{-\Phi(z)} g^{M N} v(z) \operatorname{Tr}\left\{S\left(\partial_{M} \pi-\partial_{M} \phi\right)\left(\partial_{N} \pi-\partial_{N} \phi\right)\right\}
$$


i.e.,

$$
\begin{aligned}
S_{e f f}^{(S P P)}= & -\frac{4}{k} \int d^{5} x \sqrt{-g} e^{-\Phi(z)} g^{M N} v(z) S_{1}\left(\partial_{M} \psi^{a}\right)\left(\partial_{N} \psi^{b}\right) \frac{1}{\sqrt{2 n_{F}}} \operatorname{Tr}\left[T^{a} T^{b}\right] \\
& -\frac{4}{k} \int d^{5} x \sqrt{-g} e^{-\Phi(z)} g^{M N} v(z) S_{8}^{a}\left(\partial_{M} \psi^{b}\right)\left(\partial_{N} \psi^{c}\right) \operatorname{Tr}\left[T^{a} T^{b} T^{c}\right]
\end{aligned}
$$

where $\psi^{a}=\phi^{a}-\pi^{a}$. For $n_{F}=2, \operatorname{Tr}\left[T^{a} T^{b} T^{c}\right]=\frac{i}{4} \varepsilon^{a b c}$ and the octet (triplet) part vanishes, while for $n_{F}=3$ we have:

$$
\begin{aligned}
S_{\text {eff }}^{(S P P)}= & -\frac{R^{3}}{k} \frac{2}{\sqrt{6}} \int d^{5} x \frac{1}{z^{3}} e^{-\Phi(z)} v(z) S_{1} \eta^{M N}\left(\partial_{M} \psi^{a}\right)\left(\partial_{N} \psi^{a}\right) \\
& -\frac{R^{3}}{k} d^{a b c} \int d^{5} x \frac{1}{z^{3}} e^{-\Phi(z)} v(z) S_{8}^{a} \eta^{M N}\left(\partial_{M} \psi^{b}\right)\left(\partial_{N} \psi^{c}\right) .
\end{aligned}
$$

In the Fourier space, this term involves the bulk-to-boundary propagator $S\left(\frac{q^{2}}{c^{2}}, c^{2} z^{2}\right)$ of the scalar field, together with the sources $\tilde{S}_{1(8)}$. The longitudinal part of the axial-vector field can be related to its source through the equation:

$$
\widetilde{\phi}^{a}(q, z)=\frac{1}{q^{2}} \mathcal{A}_{\|}\left(\frac{q^{2}}{c^{2}}, c^{2} z^{2}\right)\left(-i q^{\mu} \tilde{A}_{\|_{0} \mu}^{a}(q)\right),
$$

while for the combination $\widetilde{\psi}^{a}=\widetilde{\phi}^{a}-\widetilde{\pi}^{a}$ the equation involves the propagator $\Psi$ :

$$
\widetilde{\psi}^{a}(q, z)=\frac{1}{q^{2}} \Psi\left(\frac{q^{2}}{c^{2}}, c^{2} z^{2}\right)\left(-i q^{\mu} \tilde{A}_{\|_{0} \mu}^{a}(q)\right) .
$$

The contribution of only the pseudo-Goldstone bosons is selected by the condition:

$$
\widetilde{\psi}_{P}^{a}(q, z)=\frac{1}{q^{2}} \Psi\left(0, c^{2} z^{2}\right)\left(-i q^{\mu} \tilde{A}_{\|_{0} \mu}^{a}(q)\right) .
$$

From eq. (29) the condition $\partial_{z} \widetilde{\pi}^{a}=0$ holds at $q^{2}=0$ and the equation for $\Psi\left(0, c^{2} z^{2}\right)$ :

$$
\partial_{z}\left[\frac{e^{-\Phi}}{z} \partial_{z} \Psi\left(0, c^{2} z^{2}\right)\right]-\frac{g_{5}^{2} R^{2} v(z)^{2} e^{-\Phi}}{z^{3}} \Psi\left(0, c^{2} z^{2}\right)=0
$$

coincides with the equation holding for $\mathcal{A}\left(0, c^{2} z^{2}\right)$ which appears in the relation $\widetilde{A}_{\perp \mu}^{a}(0, z)=$ $\mathcal{A}\left(0, c^{2} z^{2}\right) \widetilde{A}_{\perp 0 \mu}^{a}(0)$. We can then identify $\Psi\left(0, c^{2} z^{2}\right)=\mathcal{A}\left(0, c^{2} z^{2}\right)$ [32], so that:

$$
\widetilde{\psi}_{P}^{a}(q, z)=\frac{1}{q^{2}} \mathcal{A}\left(0, c^{2} z^{2}\right)\left(-i q^{\mu} \tilde{A}_{\|_{0} \mu}^{a}(q)\right) .
$$


In this way, the $S_{\text {eff }}^{(S P P)}$ term in (42), considering only the octet contribution, reads:

$$
\begin{aligned}
i S_{\text {eff }}^{(S P P)}= & -\frac{i}{k} d^{a b c} \int \frac{d^{4} q_{1} d^{4} q_{2} d^{4} q_{3}}{(2 \pi)^{12}}(2 \pi)^{4} \delta^{4}\left(q_{1}+q_{2}+q_{3}\right) \times \\
& \int_{0}^{\infty} d z \frac{R^{3}}{z^{3}} e^{-\Phi(z)} v(z) S\left(\frac{q_{1}^{2}}{c^{2}}, c^{2} z^{2}\right) \tilde{S}_{8_{0}}^{a}\left(q_{1}\right)\left[\left(\partial_{z} \mathcal{A}\left(0, c^{2} z^{2}\right)\right)^{2}-q_{2} \cdot q_{3} \mathcal{A}\left(0, c^{2} z^{2}\right)^{2}\right] \times \\
& \left(-\frac{i}{q_{2}^{2}} q_{2}^{\mu} \tilde{A}_{\|_{0} \mu}^{b}\left(q_{2}\right)\right)\left(-\frac{i}{q_{3}^{2}} q_{3}^{\nu} \tilde{A}_{\|_{0} \nu}^{c}\left(q_{3}\right)\right) .
\end{aligned}
$$

This interaction term allows to compute the scalar couplings to pseudoscalar states. Indeed, on the basis of the AdS/CFT correspondence, the QCD three-point correlation function involving two pseudoscalar and one scalar operator:

$$
\Pi_{Q C D \alpha \beta}^{a b c}\left(p_{1}, p_{2}\right)=i^{2} \int d^{4} x_{1} d^{4} x_{2} e^{i p_{1} \cdot x_{1}} e^{i p_{2} \cdot x_{2}}\left\langle 0\left|T\left[\mathcal{O}_{5_{\alpha}}^{b}\left(x_{1}\right) \mathcal{O}_{S}^{a}(0) \mathcal{O}_{5_{\beta}}^{c}\left(x_{2}\right)\right]\right| 0\right\rangle
$$

can be obtained by functional derivation of (49) with respect to the source fields $\tilde{A}_{\|_{0}}\left(p_{1}\right)$, $\tilde{A}_{\|_{0}}\left(p_{2}\right)$ and $\tilde{S}_{8_{0}}^{a}(q)$, with the result:

$$
\begin{aligned}
& \Pi_{A d S \alpha \beta}^{a b c}\left(p_{1}, p_{2}\right)= \\
& \frac{p_{1 \alpha} p_{2 \beta}}{p_{1}^{2} p_{2}^{2}} \frac{2 R^{3}}{k} d^{a b c} \int_{0}^{\infty} d z \frac{1}{z^{3}} e^{-\Phi} v(z) S\left(\frac{q^{2}}{c^{2}}, c^{2} z^{2}\right)\left[\left(\partial_{z} \mathcal{A}\left(0, c^{2} z^{2}\right)\right)^{2}-\frac{q^{2}}{2} \mathcal{A}\left(0, c^{2} z^{2}\right)^{2}\right]
\end{aligned}
$$

with $q=-\left(p_{1}+p_{2}\right)$. The AdS expression of the strong SPP couplings follows writing the bulk-to-boundary propagator $S$ in terms of the scalar mass poles, of the residues and of the normalizable eigenfunction $\tilde{S}_{n}\left(\hat{z}^{2}\right)$ in (17). Using the integral representation of the Tricomi function [29, 33]:

$$
U(a, b, x)=\frac{1}{\Gamma(a)} \int_{0}^{1} d y \frac{y^{a-1}}{(1-y)^{b}} \exp \left[-\frac{y}{1-y} x\right]
$$

one derives the generating function of the Laguerre polynomials [14]:

$$
\frac{1}{(1-y)^{2}} \exp \left[-\frac{y}{1-y} x\right]=\sum_{n=0}^{\infty} L_{n}^{1}(x) y^{n}
$$

so that:

$$
S\left(\frac{q^{2}}{c^{2}}, c^{2} z^{2}\right)=\frac{1}{R c} \sqrt{\frac{8}{N_{c}}} \pi \sum_{n=0}^{\infty} \frac{F_{n} \tilde{S}_{n}\left(c^{2} z^{2}\right)}{q^{2}+m_{n}^{2}+i \varepsilon}
$$


Moreover, defining the scalar form factor $F_{P}$ :

$$
\left\langle P^{d}\left|\mathcal{O}_{S}^{a}\right| P^{e}\right\rangle=F_{P}^{d a e}\left(q^{2}\right)
$$

we have:

$$
\Pi_{Q C D \alpha \beta}^{a b c}\left(p_{1}, p_{2}\right)=-\frac{p_{1 \alpha} p_{2 \beta}}{p_{1}^{2} p_{2}^{2}} f_{\pi}^{2} F_{P}^{a b c}\left(q^{2}\right) .
$$

The AdS expressions of the scalar form factor and of the $g_{S_{n} P P}$ couplings follow:

$$
\begin{aligned}
F_{P}^{a b c}\left(q^{2}\right) & =-d^{a b c} \frac{1}{k} \frac{2}{f_{\pi}^{2}} \int_{0}^{\infty} d z \frac{R^{3}}{z^{3}} e^{-\Phi} v(z) S\left(\frac{q^{2}}{c^{2}}, c^{2} z^{2}\right)\left[\left(\partial_{z} \mathcal{A}\left(0, c^{2} z^{2}\right)\right)^{2}-\frac{q^{2}}{2} \mathcal{A}\left(0, c^{2} z^{2}\right)^{2}\right] \\
& =-d^{a b c} \sum_{n=0}^{\infty} \frac{F_{n} g_{S_{n} P P}}{q^{2}+m_{n}^{2}}
\end{aligned}
$$

with

$$
g_{S_{n} P P}=\frac{1}{k} \frac{2}{f_{\pi}^{2}} \int_{0}^{\infty} d z \frac{R^{3}}{z^{3}} e^{-\Phi} v(z) \frac{1}{R c} \sqrt{\frac{8}{N_{c}}} \pi \tilde{S}_{n}\left(c^{2} z^{2}\right)\left[\left(\partial_{z} \mathcal{A}\left(0, c^{2} z^{2}\right)\right)^{2}+\frac{m_{S_{n}}^{2}}{2} \mathcal{A}\left(0, c^{2} z^{2}\right)^{2}\right]
$$

To compute $g_{S_{n} P P}$ from (58), one needs $\mathcal{A}\left(0, c^{2} z^{2}\right)$, which can be obtained solving (27). However, since $v(z)$ is small (it depends on $m_{q} / R$ ), one can neglect terms proportional to $v^{2}$ and identify $\mathcal{A}\left(0, \hat{z}^{2}\right)$ with $\mathcal{A}^{(0)}\left(0, \hat{z}^{2}\right)$ solution of:

$$
\partial_{\hat{z}}\left(\frac{e^{-\hat{z}^{2}}}{\hat{z}} \partial_{\hat{z}} \mathcal{A}^{(0)}\left(0, \hat{z}^{2}\right)\right)=0
$$

with $\mathcal{A}^{(0)}\left(0, \hat{z}^{2}\right) \underset{z \rightarrow 0}{\rightarrow} 1$. The regular solution is $\mathcal{A}^{(0)}\left(0, \hat{z}^{2}\right)=1$.

The expression of $g_{S_{0} P P}$ for the lowest radial number $n=0$, since $\tilde{S}_{0}\left(\hat{z}^{2}\right)=\sqrt{2} \hat{z}^{3}$, is:

$$
g_{S_{0} P P}=\frac{\sqrt{N_{c}}}{4 \pi} \frac{m_{S_{0}}^{2}}{f_{\pi}^{2}} R c \int_{0}^{\infty} d \hat{z} e^{-\hat{z}^{2}} v(\hat{z}) .
$$

The coupling depends linearly on the field $v$. The numerical result is small, of the order of 10 $\mathrm{MeV}$ depending on the quark mass used as an input. On the other hand, phenomenological determinations of the SPP couplings indicate sizeable values, showing that the scalar states are characterized by their large couplings to light pseudoscalar mesons. For example, the experimental value of $g_{a_{0} \eta \pi}$ is: $g_{a_{0} \eta \pi}=12 \pm 6 \mathrm{GeV}$, while for $f_{0}$ the result of a QCD estimate is: $g_{f_{0} K^{+} K^{-}} \simeq 6-8 \mathrm{GeV}$ [34]. The origin of the small value for the $S P P$ couplings in the AdS/QCD soft-wall model can be traced to the expression of $v$ which is determined by 
the light quark mass (larger results would be obtained, e.g., using $v$ computed in the hardwall approach). The drawback confirms the difficulty of the soft-wall model in correctly describing chiral symmetry breaking; it could be probably avoided including potential terms in the effective action (3), a possibility which deserves a dedicated study.

\section{CONCLUSIONS}

We have studied the scalar sector in the $5 d$ AdS soft-wall model proposed as a QCD dual, finding that the masses and decay constants of scalar mesons are close to experiment and QCD determinations. The two-point correlation function of the scalar operator has a power expansion similar to QCD, with violations in the dimension six condensates computed assuming factorization. A dimension two condensate term, absent in QCD, appears in the power expansion of the AdS expression, analogously to the two-point correlators of vector meson and scalar glueball operators. The strong couplings of scalar states to pairs of light pseudoscalar mesons are smaller than in phenomenological determinations, as a consequence of the difficulty of correctly describing chiral symmetry breaking within this model. This difficulty could be avoided including additional potential terms in the effective Lagrangian defining the model.

\section{Acknowledgments.}

This paper is dedicated to the memory of Beppe Nardulli.

We are grateful to M. Pellicoro for discussions. This work was supported in part by the EU Contract No. MRTN-CT-2006-035482, "FLAVIAnet".

[1] J. M. Maldacena, Adv. Theor. Math. Phys. 2, 231 (1998) [Int. J. Theor. Phys. 38, 1113 (1999)]; E. Witten, Adv. Theor. Math. Phys. 2, 253 (1998); S. S. Gubser, I. R. Klebanov and A. M. Polyakov, Phys. Lett. B 428, 105 (1998).

[2] E. Witten, Adv. Theor. Math. Phys. 2, 505 (1998).

[3] For a recent review see: J. Erdmenger, N. Evans, I. Kirsch and E. Threlfall, Eur. Phys. J. A 35, 81 (2008) and references therein. 
[4] J. Polchinski and M. J. Strassler, Phys. Rev. Lett. 88, 031601 (2002).

[5] A discussion about the conformal window of QCD can be found in S. J. Brodsky and G. F. de Teramond, arXiv:0802.0514 [hep-ph] and references therein.

[6] J. Erlich, E. Katz, D. T. Son and M. A. Stephanov, Phys. Rev. Lett. 95, 261602 (2005).

[7] L. Da Rold and A. Pomarol, Nucl. Phys. B 721, 79 (2005).

[8] S. J. Brodsky and G. F. de Teramond, Phys. Lett. B 582, 211 (2004), Phys. Rev. Lett. 94, 201601 (2005) and Phys. Rev. Lett. 96, 201601 (2006); U. Gursoy, E. Kiritsis and F. Nitti, JHEP 0802, 019 (2008).

[9] H. Boschi-Filho, N. R. F. Braga and C. N. Ferreira, Phys. Rev. D 73, 106006 (2006) [Erratumibid. D 74, 089903 (2006)] and Phys. Rev. D 74, 086001 (2006).

[10] A. Karch, E. Katz, D. T. Son and M. A. Stephanov, Phys. Rev. D 74, 015005 (2006).

[11] O. Andreev, Phys. Rev. D 73, 107901 (2006).

[12] B. Batell and T. Gherghetta, arXiv:0801.4383 [hep-ph].

[13] O. Andreev and V. I. Zakharov, Phys. Rev. D 74, 025023 (2006).

[14] H. R. Grigoryan and A. V. Radyushkin, Phys. Rev. D 76, 095007 (2007).

[15] H. J. Kwee and R. F. Lebed, JHEP 0801, 027 (2008).

[16] P. Colangelo, F. De Fazio, F. Jugeau and S. Nicotri, Phys. Lett. B 652, 73 (2007).

[17] C. A. Ballon Bayona, H. Boschi-Filho and N. R. F. Braga, JHEP 0803, 064 (2008).

[18] H. J. Kwee and R. F. Lebed, Phys. Rev. D 77, 115007 (2008).

[19] L. Da Rold and A. Pomarol, JHEP 0601, 157 (2006); T. Huang and F. Zuo, arXiv:0708.0936 [hep-ph].

[20] A. Vega and I. Schmidt, arXiv:0806.2267 [hep-ph].

[21] I. R. Klebanov and E. Witten, Nucl. Phys. B 556, 89 (1999).

[22] P. Colangelo, F. De Fazio, F. Jugeau and S. Nicotri, arXiv:0711.4747 [hep-ph].

[23] Drawbacks concerning the scale dependence of the chiral condensate are discussed in A. Cherman, T. D. Cohen and E. S. Werbos, arXiv:0804.1096 [hep-ph].

[24] L. J. Reinders, H. Rubinstein and S. Yazaki, Phys. Rept. 127, 1 (1985).

[25] A. Gokalp, Y. Sarac and O. Yilmaz, Eur. Phys. J. C 22, 327 (2001).

[26] F. De Fazio and M. R. Pennington, Phys. Lett. B 521, 15 (2001).

[27] P. Colangelo and A. Khodjamirian, in 'At the Frontier of Particle Physics / Handbook of QCD', ed. by M. Shifman (World Scientific, Singapore, 2001) vol. 3* 1495-1576. 
[28] O. Andreev, Phys. Rev. D 73, 107901 (2006).

[29] F. Zuo and T. Huang, arXiv:0801.1172 [hep-ph].

[30] H. Forkel, arXiv:0711.1179 [hep-ph].

[31] A recent discussion and references can be found in: V. I. Zakharov, "Old problems of QCD phenomenology in view of AdS/QCD correspondence", Proceedings of QCD@Work 2007, International Workshop on Quantum Chromodynamics Theory and Experiment, Martina Franca, Italy, 16-20 June 2007, P. Colangelo et al. editors, AIP Conf. Proc. 964, 143 (2007).

[32] H. R. Grigoryan and A. V. Radyushkin, Phys. Rev. D 76, 115007 (2007).

[33] A. Erdélyi, Higher Trascendental Functions, Vol. 1 (New York-Toronto-London) (1953).

[34] P. Colangelo and F. De Fazio, Phys. Lett. B 559, 49 (2003). 